\title{
Stadtstruktur und Kriminalität
}

\section{Dietrich Oberwittler}

Max-Planck-Institut für ausländisches und internationales Strafrecht, Abt.

Kriminologie, Freiburg

erscheint in:

Pöge, Andreas / Hermann, Dieter (Hg.) (2018):

Kriminalsoziologie. Handbuch für Wissenschaft und Praxis, BadenBaden: Nomos Verlag, S. 317-336

1. Auflage 2018, ISBN print: 978-3-8487-2806-0, ISBN online: 978-3-8452-7184-2 DOI: $10.5771 / 9783845271842-316$

\section{d.oberwittler@mpicc.de}

https://www.mpicc.de/de/home/oberwittler.html 


\section{Stadtstruktur und Kriminalität}

\section{Dietrich Oberwittler}

\section{Einleitung}

Städte sind nicht nur wirtschaftliche, kulturelle und politische Zentren, sondern gelten häufig auch Zentren von Kriminalität und Gewalt. Deutsche Städte wie Frankfurt oder Köln, auch als „Chicago am Rhein“ tituliert (Müller/Mueller 2011), oder das US-amerikanische ,Original “ haben sich über Jahrzehnte einen Ruf als Kriminalitätshochburgen erworben. Doch weder im historischen noch im internationalen Vergleich gesehen ist es gerechtfertigt, Städte als ,natürliche‘ Kriminalitätsschwerpunkte anzusehen. Noch im 19. Jahrhundert war die Gewaltkriminalität in vielen ländliche Regionen Mittel- und Westeuropas höher als in den Städten, welche eine Vorreiterrolle im Zivilisierungsprozess spielten (Eisner 1997; Pinker 2011; Thome 2002), und auch heute liegen die Mordraten der größten Städte etwa in Finnland und Osteuropa unterhalb des nationalen Niveaus (Savolainen et al. 2008; United Nations Office on Drugs and Crime 2013, S. 28).

Ein Blick auf die Polizeiliche Kriminalstatistik zeigt, dass die deutschen Großstädte nicht dramatisch stärker mit registrierter Gewalt- und Drogenkriminalität belastet sind als Deutschland insgesamt. In den Abbildungen 1a und 1b ist die relative Höhe der Häufigkeitsziffern (HZ) einiger Gewaltdelikte für die Städte unterschiedlicher Größenklassen im Vergleich zur nationalen $\mathrm{HZ}$ graphisch dargestellt. Der Wert 1,5 beispielsweise zeigt an, dass die HZ in Städten um 50 \% höher ist als die nationale HZ. Während Tötungsdelikte in den Städten heute (im Gegensatz zu vergangenen Jahrzehnten) nicht häufiger sind als im nationalen Durchschnitt, kommen Vergewaltigungen, gefährliche und schwere Körperverletzungen und vor allem Raubdelikte in Städten zwischen 100.000 und 500.000 Einwohnern etwa um den Faktor 1,5 und in den 14 größten Städten ab 500.000 Einwohnern etwa um den Faktor 1,5 bis 2,0 mal häufiger vor als im nationalen Durchschnitt. Dabei ist für die größten Städte seit 1995 bei allen Delikten ein rückläufiger Trend sichtbar, während der Trend bei den mittelgroßen Städten leicht ansteigend ist. Der sinkende Trend in den größten Städten könnte mit der deutlich fallenden 
Gewaltkriminalität in hochentwickelten Ländern insgesamt zusammenhängen (Farrall et al. 2014). Die größten deutschen Städte sind heute weniger als noch vor einer Generation als besondere Gewaltorte anzusehen.

Abb. 1a: Kriminalitätsbelastung von Städten mit 100.000 bis 500.000 Einwohnern im Vergleich zu Deutschland insgesamt, 1955 bis 2015 (Häufigkeitsziffer Städte / Bundesrep. Deutschland)

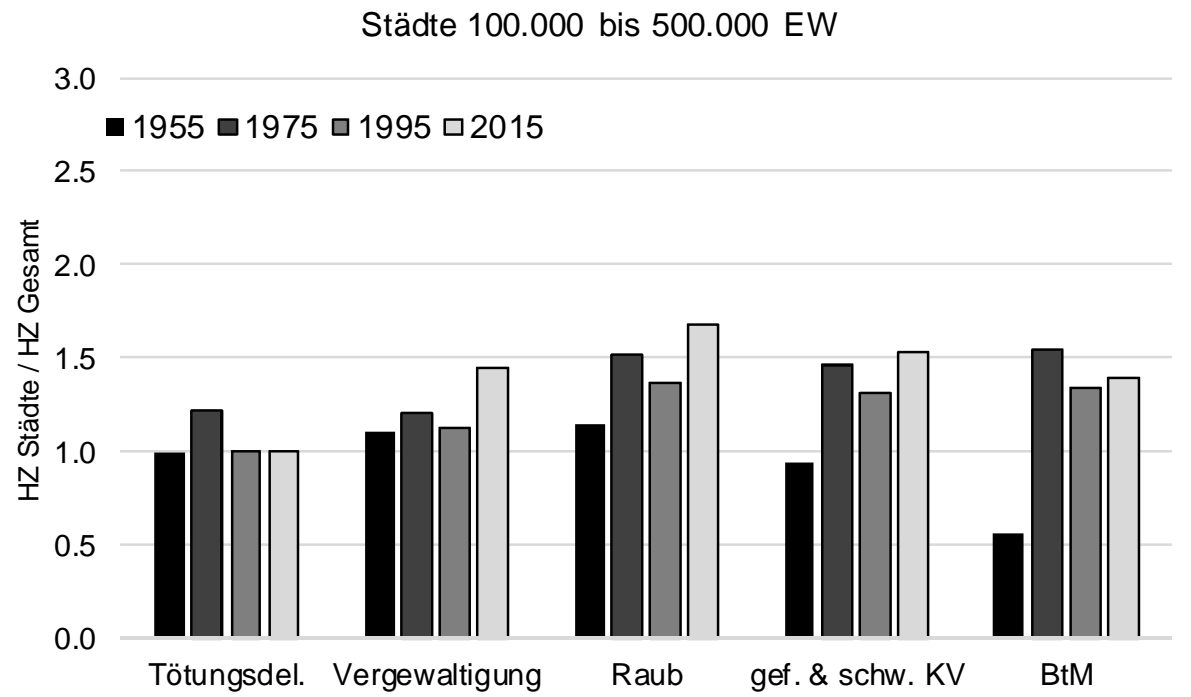

Daten: Bundeskriminalamt, Polizeiliche Kriminalstatistik, verschiedene Bände, eigene Berechnung

Abb. 1b: Kriminalitätsbelastung von Städten über 500.000 Einwohnern im Vergleich zu Deutschland insgesamt, 1955 bis 2015 (Häufigkeitsziffer Städte / Bundesrep.

Deutschland) 


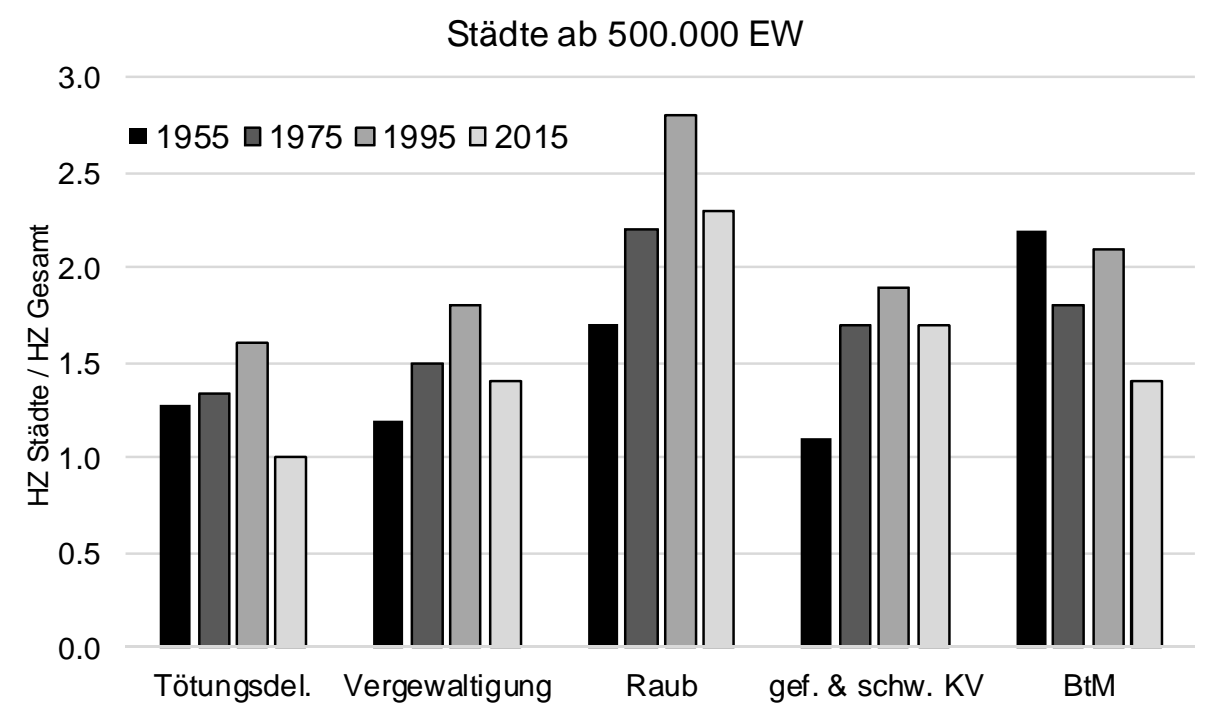

Daten: Bundeskriminalamt, Polizeiliche Kriminalstatistik, verschiedene Bände, eigene Berechnung

Jedoch sind es in erster Linie die enormen Unterschiede innerhalb der Städte und die Konzentration auf wenige Stadtviertel und ,Mikro-Räume', die das Erscheinungsbild der Kriminalität in Städten prägen, und die die größte Aufmerksamkeit der Forschung hervorrufen. Zusammenhänge von Stadtstruktur und Kriminalität haben die Soziologie seit ihrer Entstehung beschäftigt und entscheidend zur Theoriebildung und empirischen Forschung zu den Ursachen von Kriminalität beigetragen. Dabei stehen „klassische“ Formen der Kriminalität mit Verankerung in Raum und Zeit und klaren Opfern - z.B. Sachbeschädigung, Diebstahl, Wohnungseinbruch, Raub, körperliche Gewalt - im Vordergrund, während die zunehmende Internetkriminalität von geographischen Räumen weitgehend abgelöst ist. Aber alltägliche Erfahrungen von und Befürchtungen vor Wohnungseinbrüchen, Überfällen oder Belästigungen werden auch in der Zukunft die Raumwahrnehmungen der Menschen beeinflussen und über die Attraktivität und Lebensqualität von Wohngebieten mitentscheiden. Städtische Sicherheit und die Frage nach geeigneter Prävention hat sich zu einem wichtigen Politikfeld entwickelt (Frevel 2012). 
Industrialisierung und Urbanisierung im 19. Jahrhundert ebenso wie Strukturwandel und Deindustrialisierung seit den 1970er Jahren haben im Zusammenhang von sozialer und ethnischer Segregation städtische ,Problemgebiete` entstehen lassen, die seit den klassischen Beschreibungen der Elendsviertel im frühindustriellen England von Friedrich Engels (1845) und Henry Mayhew (1861) Gegenstand von Sozialforschung sind. Die stadtsoziologische ,Chicago School' entwickelte in den 1930er und 1940er Jahren die Theorie der sozialen Desorganisation (Shaw/McKay 1969 [1942]), die benachteiligte Wohnquartiere als problematische Entwicklungsund Handlungskontexte ihrer (jugendlichen) Bewohner sieht, und prägte bis heute eine wichtige kriminalsoziologische Forschungsrichtung. Die Desorganisationstheorie wie auch andere ,klassische` Erklärungsansätze der Kriminalsoziologie sieht Kriminalität als Folge sozialer Missstände. Eine deutlich jüngere und in den letzten Jahren stark expandierende Forschungsrichtung versteht Kriminalität dagegen eher als Begleiterscheinung des modernen Lebens und Ergebnis rationalen Handelns und interessiert sich für städtische Gelegenheitsstrukturen als Kontexte krimineller Ereignisse. Obwohl beide Perspektiven für das Verständnis von urbanen Kriminalitätsphänomenen berechtigt sind und sich ergänzen könnten, sind integrative Forschungsansätze noch selten. Ihre gemeinsame Grundannahme ist, dass Stadtstrukturen eine eigenständige kausale Rolle in der Entstehung von Kriminalität haben. Den geographischen Raumeinheiten werden Merkmale zugeschrieben, die sich entweder aus der sozialen Zusammensetzung und den wechselseitigen Beziehungen der Menschen, die sich in ihnen aufhalten, entwickeln, oder sich aus physischen und städtebaulichen Eigenschaften, der geographischen Lage, der Verkehrswege usw. ergeben. Das macht Erklärungen und empirische Studien besonders komplex, denn die kollektive Ebene von Stadtvierteln und Mikro-Räumen ersetzt die individuelle Ebene der handelnden Menschen nicht, sondern ergänzt sie. Um zu verstehen, wie Menschen in ihrem Handeln durch räumliche Kontexte beeinflusst werden, und wie aus der Summe vieler individueller Handlungen kollektive Phänomene entstehen, greifen viele soziologische Analysen auf ein allgemeines Modell der Makro-Mikro-MakroVerbindungen, das sog. ,Coleman-Boot‘, zurück (Coleman 1990; Opp 2015).

In diesem Beitrag sollen empirische Erkenntnisse zur kleinräumigen Verteilung von Kriminalität in Großstädten und die maßgeblichen theoretischen Ansätze zu ihrer Erklärung vorgestellt werden. Die Forschung dazu hat in den letzten zwanzig Jahren enorm zugenommen. 
Ausführlichere Überblicke bieten u.a. Baumer/Arnio (2015), Bottoms (2012), Oberwittler (2013),

Sampson et al. (2002) und Weisburd et al. (2016). Während sich deutsche Forscher in den 1960er und 1970er Jahren mit einigen wegweisenden Studien an diesem hierzulande häufig als Kriminalgeographie benannten Gebiet beteiligt haben (Frehsee 1979; Opp 1968; Schwind et al. 1978), findet der größte Teil der neueren Forschung seither in den angelsächsischen Ländern und den Niederlanden statt.

\section{Empirische Zusammenhänge von Stadtstruktur und Kriminalität}

$\mathrm{Zu}$ den häufigsten und robustesten Befunden von Analysen innerstädtischer

Kriminalitätsverteilungen gehört der enge Zusammenhang mit Indikatoren sozialer

Benachteiligung wie Arbeitslosigkeit oder Einkommensarmut. Dies gilt sowohl für europäische Städte wie Stockholm (Wikström 1991), Basel (Eisner 2001) oder Köln (Opp 1968, Oberwittler 2001) als auch für US-amerikanische Städte (Chamberlain/Hipp 2015; Pratt/Cullen 2005).

Häufig werden dabei Armutsindikatoren mit dem Bevölkerungsanteil ethnischer Minderheiten zu einem Index zusammengezogen, da soziale und ethnische Segregation sehr eng verknüpft und statistisch kaum zu trennen sind. Dies gilt ganz besonders für die Armenghettos der Afroamerikaner und Latinos in den USA, in denen sich Gewalt- und Bandenkriminalität konzentrieren (Crutchfield 2015; Peterson/Krio 2010; Wacquant 2009). Längsschnittstudien haben jedoch gezeigt, dass der Zusammenhang von Benachteiligung und Kriminalität nicht einseitig ist, sondern Kriminalität im Zeitverlauf zu einer Verschärfung sozialstruktureller Benachteiligung führt, unter anderem durch selektive Abwanderung derer, die es sich leisten 
können (Hermann/Laue 2003; Hipp 2010; Hipp/Steenbeek 2016; Hipp/Wickes 2016; Morenoff/Sampson 1997).

Anhänger des in der deutschen Kriminalsoziologie besonders verankerten Definitionsansatzes haben die Verwendung polizeilicher Kriminalitätsdaten kritisiert, weil damit eher eine selektive und verzerrende Registrierung von Unterschichtsangehörigen und Minderheiten als eine wirkliche Belastung mit strafbaren Handlungen gemessen werde (Belina/Wehrheim 2011; Busch/Werkentin 1992). Jedoch zeigen Studien, die auf Opfer- und Täterbefragungen basieren, dass sozialräumliche Zusammenhänge zwischen sozialstruktureller Benachteiligung und Kriminalität auch im Dunkelfeld der Kriminalität vorhanden und z.T. recht stark sind (Estrada/Nilson 2008; Lauritsen 2001; Oberwittler 2004c; Sampson et al 1997; Tseloni 2006; Wikström et al. 2012, S. 232, 327).

Aber nicht nur sozial benachteiligte Wohngebiete, sondern auch innenstadtnahe Räume mit einer hohen Dichte an Geschäften und Gaststätten sind Kriminalitätsschwerpunkte und weisen oft die höchste Gewaltbelastung in Großstädten auf (Ceccato/Oberwittler 2008; Groff/Lockwood 2014; Roncek/Maier 1991). In lediglich etwa $3 \%$ bis $5 \%$ der Straßenabschnitte konzentrieren sich bis zu $50 \%$ der Tatorte urbaner Gewaltkriminalität (Sherman et al. 1989). Diese Erkenntnis hat für viele Forscher die Perspektive auf die Entstehungsbedingungen von Gewalt verändert und die Rolle der Gelegenheitsstrukturen in den Vordergrund gerückt (s.u.).

Die geographische Verteilung von Kriminalität verdeutlicht unmittelbar die Bedeutung von Mobilität sowohl innerhalb der Stadt als auch über die Stadtgrenzen hinaus. Tatorte sind ganz überwiegend nicht identisch mit den Wohnadressen von Tätern und Opfern, sodass sich die räumlichen Muster und Zusammenhänge je nach Perspektive unterscheiden. Die Zentrums-

funktion von Städten zieht viele Menschen von außerhalb an, die im Zuge ihrer Alltagsaktivitäten wie Arbeiten, Einkaufen oder Ausgehen Täter oder Opfer werden. In badenwürttembergischen Großstädten kommen zwischen einem Drittel und der Hälfte der Tatverdächtigen aus anderen Gemeinden (Oberwittler/Gerstner 2011, S. 23), und Täter und 
Opfer von Gewaltkriminalität in der Kölner Altstadt sind sogar zu 80 \% Auswärtige (Oberwittler 2004b). Es wäre daher sinnvoller, die räumliche Konzentration von Tatorten an der zugrundeliegenden Aufenthaltsbevölkerung zu messen. Da diese jedoch nicht bekannt ist, sind nicht nur die gängigen, an der Wohnbevölkerung standardisierten Häufigkeits- und Belastungsziffern der Kriminalität irreführend, sondern auch auf solchen Raten basierenden Zusammenhangsanalysen mit sozialräumlichen Bedingungen (Oberwittler 2004b).

\section{Erklärungsansätze der Zusammenhänge von Stadtstruktur und Kriminalität}

Kriminalsoziologische Erklärungsansätze versuchen theoriegeleitete Antworten auf die Frage nach den Ursachen von Kriminalität zu geben. Bezogen auf räumliche Verteilungen von Kriminalität bedeutet dies, nach den sozialräumlichen und geographischen Wirkungsmechanismen zu suchen, die Kriminalität über die individuellen Ursachenfaktoren hinaus fördern oder behindern. Dabei ist wichtig, dass Stadtviertel keine Inseln sind, sondern Teile eines durch hohe Mobilität verbundenen räumlichen Systems.

Die Erklärungsansätze können anhand der beiden grundsätzlichen Perspektiven auf die sozialräumlichen Bedingungen sortiert werden, die die kriminellen Neigungen von Individuen fördern, oder aber die Bedingungen, die kriminelle Handlungen befördern. Die erste Perspektive sieht städtische Wohngebiete als längerfristige Entwicklungskontexte (vor allem für Kinder und Jugendliche), die zweite Perspektive sieht städtische Räume ganz allgemein - unabhängig vom Wohnsitz - als Ereigniskontexte (Wikström/Sampson 2003). In den beiden Perspektiven stehen jeweils andere Wirkungsmechanismen im Vordergrund, auch wenn es große Überlappungen gibt, und verschiedene Kontexte durch Mobilität stets miteinander verbunden sind. Eine isolierte Betrachtung jeweils einer Perspektive würde die Gefahr einer ,Balkanisierung ' des Forschungsfeldes in sich bergen (Taylor 2015, S. 2).

Auch die Mobilität zwischen Wohnadresse und Tatort ist Gegenstand der Forschung zu urbaner Kriminalität. Patricia und Paul Brantingham ( 2008, 2013) beschreiben in der ,Crime Pattern Theory 'typische Mobilitätsmuster in Städten, die Täter und Opfer im Verlauf ihrer Alltagsroutinen an bestimmten Knotenpunkten zusammenbringen, welche günstige Situationen für Kriminalität bieten - häufig an oder in der Nähe von Haltestellen des öffentlichen Verkehrs. 
Das ,Distance-Decay “ Modell zeigt auf, dass Täter häufig zwar nicht in der unmittelbaren Umgebung ihrer Wohnung, aber doch mit größerer Wahrscheinlichkeit in geringerer Entfernung Straftaten begehen (Andresen 2014, S. 53; Wikström et al. 2012, S. 239). Diese Regel gilt nicht gleichermaßen für unterschiedliche Delikte, z.B. eher für Gewaltdelikte und weniger für Ladendiebstahl.

\subsection{Stadtstruktur als Entwicklungskontext ${ }^{1}$}

Führt die Konzentration sozialer Benachteiligungen in Wohnquartieren zu einer Verstärkung von problematischen Verhaltensweisen und Entwicklungstendenzen von Kindern und Jugendlichen (im Folgenden vereinfacht Jugendliche), die in diesen Quartieren wohnen? Diese Kernfrage wird nicht nur im Hinblick auf Delinquenz und Kriminalität, sondern auch auf Bildungsleistungen, Arbeitsmarkterfolg und Gesundheit untersucht (vgl. allgemein Chang et al. 2016; Nieuwenhuis/Hooimeijer 2016; Sampson et al. 2002).

Für die Erklärung von Kontexteffekten auf abweichendes Verhalten von Jugendlichen ist die Theorie der sozialen Desorganisation von Shaw und McKay (1969 [1942]) bis heute maßgeblich geblieben. Dies liegt auch daran, dass Shaw und McKay mehrere Wirkungsmechanismen postuliert haben: Einerseits die informellen Sozialkontrolle der (erwachsenen) Bewohner über Jugendliche, der im „systemischen Modell“ und im Ansatz der kollektiven Wirksamkeit die entscheidende Rolle spielt (s.u.), andererseits aber auch die Kontakte zwischen delinquenten Jugendlichen, den sie sich als einen Prozess der , cultural transmission ' devianter Normen und Verhaltensmuster von einer Generation von Jugendlichen auf die jeweils nachwachsende Generation vorstellten (Shaw/McKay 1969 [1942], S. 168). Dieser Wirkungsmechanismus der Gleichaltrigeneinflüsse ist erst seit einigen Jahren wieder stärker in den Vordergrund gerückt und wird mit der Methode der Netzwerkanalyse intensiv erforscht (Beier 2014, 2016; Dishion/Tipsord 2011, Gerstner/Oberwittler 2015; Harding 2009; Haynie/Kreager 2013; Knecht

${ }^{1}$ Dieser Abschnitt basiert auf Oberwittler $(2013,2017)$. 
et al 2010; Weerman 2011). Der Mechanismus der sozialräumlichen Verstärkung von Jugenddelinquenz durch Gleichaltrige ist mit Krankheitsepidemien verglichen worden: Wenn subkulturelle Einstellungen in einem sozial segregierten Wohnquartier durch die Konzentration benachteiligter Familien Fuß fassen, könnte der Kontakt zwischen den Jugendlichen deviante Normen verbreiten und verstärken (Crane 1991). Die Anwesenheit vieler Jugendlicher mit delinquenten Neigungen stellt auch einen situativen Faktor für Delinquenz dar, denn Jugendliche begehen Delikte ganz überwiegend in Gemeinschaft mit anderen (Wikström et al. 2012, S. 329). Jugendgangs als eine mehr oder weniger organisierte Form von Jugenddelinquenz haben meist einen starken territorialen Bezug und bewirken bei ihren Mitgliedern eine deutliche Intensivierung ihrer Delinquenz (Klein et al. 2006; Pitts 2008).

\section{US-amerikanische Studien}

Empirische Untersuchungen vor allem aus den USA haben die Existenz von sozialräumlichen Kontexteffekten auf Jugenddelinquenz bestätigt, während europäische Studien weniger eindeutig sind. Die bedeutendsten US-amerikanischen Studien sind die „National Longitudinal Study of Adolescent Health“ (Add Health) mit ca. 20.000 Befragten und vier Befragungswellen über mehr als zehn Jahre, das „Project of Human Development in Chicago Neighbourhoods“ (PHDCN) mit ca. 6.000 Befragten und drei Befragungswellen, sowie die experimentelle Studie „Moving to Opportunity“ (MTO).

In der Chicagoer Längsschnittstudie erklärte die sozialräumliche Benachteiligung der Wohnquartiere $30 \%$ bis $60 \%$ der Höherbelastung der schwarzen Jugendlichen gegenüber den weißen Jugendlichen mit selbstberichteter Gewaltdelinquenz (Kirk 2008; Sampson et al. 2005). Der in den USA bei der Erklärung von Gewalt oft im Vordergrund stehende ,Race'-Faktor ent- 
puppt sich demnach zumindest teilweise als eine Folge sozialräumlicher Benachteiligungen. Auf der Basis derselben Chicagoer Daten zeigten andere Auswertungen eine signifikante Verschlechterung von Schulleistungen, wenn sich wenige Tage zuvor in unmittelbarer Nähe ein Mord ereignet hatte (Sharkey 2010) und eine Verdoppelung der Wahrscheinlichkeit, schwere Gewalt auszuüben, wenn die Jugendlichen bis zu zwei Jahren zuvor Zeuge von Gewalt mit Schusswaffen geworden waren (Bingenheimer et al. 2005).

Auch die ,Add Health'-Studie kann die Existenz von sozialräumlichen Verstärkungseffekten belegen. Die Armutskonzentration des Wohngebietes hat einen signifikanten Effekt auf die Gewaltdelinquenz der Jugendlichen und führt gemeinsam mit einer Reihe weiterer Risikofaktoren dazu, dass ethnische Zuordnungen statistisch gänzlich unbedeutend werden (Bellair/McNulty 2005; De Coster et al. 2006).

Die ,Moving to Opportunity'-Studie (MTO) ist aufgrund ihrer experimentellen Anlage einmalig. Einkommensarme, meist schwarze Familien aus Wohngebieten konzentrierter Armut erhielten nach dem Zufallsprinzip Gutscheine für Umzüge in ,bessere' Wohngebiete; die weitere Entwicklung der umgezogenen Familien und einer in den ursprünglichen Wohngebieten verbliebenen Vergleichsgruppe wurde über bislang fünf Jahre verfolgt. Während die Delinquenz von Mädchen in der Experimentalgruppe insgesamt abnahm, reagierten Jungen auf den Umzug zwar mit einem Rückgang der Gewaltdelikte, jedoch gleichzeitig mit einem Anstieg der Eigentumsdelikte (Burdick-Will/Ludwig 2013; Ludwig et al. 2008). Die Forscher erklären diesen paradoxen Befund mit der erhöhten Anreizstruktur für Eigentumsdelikte in den wohlhabenden Wohngebieten.

Sehr bedeutsam sind auch Ergebnisse von Längsschnittstudien, die die Entwicklung von Kindern und Jugendlichen über viele Jahre weiterverfolgen und dabei sogar noch stärkere langfristige Effekte sozialräumlicher Benachteiligungen auf Delinquenz oder verwandte Verhaltensbereiche finden als Studien mit kurzem Zeithorizont, was mit kumulativen Verstärkungseffekten erklärt wird (Sharkey/Sampson 2010; Ludwig et al. 2012; Wodtke et al. 2011).

\section{Europäische Studien}

Die europäische Forschung zu sozialräumlichen Wirkungen auf Jugenddelinquenz ist weniger entwickelt und methodisch weniger aufwändig. Es dominierten bislang Querschnittsstudien, 
deren Ergebnisse zurückhaltender interpretiert werden müssen, und die mehrheitlich keine Kontexteffekte auf Jugenddelinquenz gefunden haben (Oberwittler 2013, S. 58). In Skandinavien können Forscher auf Bevölkerungsregister z.B. von Gemeinden, Schul- und Justizbehörden zurïckgreifen und so methodisch einzigartige Längsschnittstudien gesamter Bevölkerungsgruppen realisieren. Mehrere registerbasierte Studien in Schweden fanden nach Kontrolle der individuellen und familiären Einflussfaktoren keine sozialräumlichen Kontexteffekte (Brännström/Rojas 2012; Sarialsan et al 2013), eine dänische Studie berichtete über Langzeiteffekte des Aufwachsens in Gemeinden mit hoher Jugendkriminalität (Damm/Dustmann 2014). Als noch laufende Längsschnittstudien sind deutschen Projekte „Kriminalität in der modernen Stadt“ (CRIMOC) (Boers et al. 2010) und „Freundschaft und Gewalt im Jugendalter“ (FuGJ, Beier 2016) zu nennen, außerdem die englischen Studie PADS+ (Wikström et al. 2012, s.u.) mit Schwesterstudien in den Niederlanden und Schweden.

Eine Studie des Max-Planck-Instituts, die auf Befragungen von Jugendlichen und Bewohnern in 60 Stadtvierteln in Köln und Freiburg basiert, stellte Einflüsse des Wohngebiets für bestimmte Gruppen von Jugendlichen fest (Oberwittler 2004a, 2004c, 2007). Für einheimische Jugendliche mit auf das eigene Wohnquartier konzentriertem Freundeskreis verdoppelt sich nach die-

ser Studie beinahe die Wahrscheinlichkeit der schweren Delinquenz mit der Zunahme der sozialräumlichen Armut unter Kontrolle individueller Faktoren; gleichzeitig verliert der individuelle Arbeitslosen- bzw. Sozialhilfestatus der Eltern seine Signifikanz. Bei Mädchen nimmt insbesondere die Gewaltneigung stark zu, bei Jungen eher die Wahrscheinlichkeit schwerer Eigentumsdelikte. Ebenso wie in den erwähnten Analysen von Bellair/McNulty (2005) und De Coster et al. (2006) wirkt die sozialstrukturelle Benachteiligung also eher kollektiv über die Wohngebiete als individuell auf jugendliche Delinquenz. Allerdings gilt dies erwartungswidrig nicht für Jugendliche aus Migrantenfamilien, für die sich keine sozialräumlichen Verstärkungseffekte fanden (Oberwittler 2007). 
Angesichts der wesentlich stärkeren sozialen Ungleichheit und des niedrigeren Niveau des Wohlfahrtsstaates sind die stärkeren Belege für Stadtvierteleinflüsse in US-amerikanischen Studien nicht überraschend, sondern könnte für den Erfolg des europäischen Gesellschaftsmodells sprechen.

\section{Wechselwirkungen zwischen Kontexteinflüssen und individuellen Merkmalen}

Der Forschungsschwerpunkt hat sich in den letzten Jahren zunehmend von der pauschalen Frage nach der Existenz von Kontexteffekten auf differenzierende Fragen nach den Wechselwirkungen (oder Interaktionseffekten) individueller Jugendlicher und ihrer Familien mit den sozialräumlichen Bedingungen verlagert (Sharkey/Faber 2014; Wikström et al. 2012). Jugendliche sind keine passiven Empfänger von Einflüssen, sondern setzen sich aktiv mit ihrer sozialen Umwelt auseinander (Hurrelmann 1983). Einige Jugendliche sind empfänglich für Gefährdungen, während andere scheinbar umempfänglich (resilient) reagieren (Elliott et al. 2006). Offenbar wirken bestimmte individuelle Eigenschaften als eine Art Puffer gegen ungünstige Umwelteinflüsse.

Dieser Gedanke von Wechselwirkungen zwischen individuellen delinquenten Neigungen und situationsgebundenen Gelegenheiten steht im Zentrum der von Per-Olof Wikström formulierten ,Situational Action Theory“ (SAT) (Wikström 2006, Wikström u.a. 2012). Die SAT rückt den individuellen „Wahrnehmungs-Entscheidungs-Prozess“ für oder gegen den Normbruch ins Zentrum und fragt nach den Wechselwirkungen zwischen den Situationen, die sich durch unterschiedliche Gelegenheiten, Provokationen und Kontrollintensitäten auszeichnen, und den individuellen Neigungen für abweichendes Verhalten. Damit werden in diesem Theoriemodell Elemente von ,Rational Choice'-und ,Routine Activity'-Theorien mit ,klassischen“ täterorientierten Theorien verbunden. In empirischen Analysen der Längsschnittstudie „PADS+““ (einer Stichprobe von etwa 700 Jugendlichen in Peterborough/Großbritannien) bestätigten sich die theoretischen Annahmen der SAT. Ein besonderes Element der „PADS+“-Studie ist die detaillierte Erfassung der Aufenthaltsorte und Aktivitäten der Jugendlichen über mehrere Tage, sodass eine wesentlich präzisere Messung der Situationen möglich wird, in denen Jugendliche delinquente Handlungen begehen. Die Ergebnisse zeigen, dass Delinquenz ist am wahrscheinlichsten ist, wenn sich Jugendliche mit delinquenten Verhaltensdispositionen an 
Orten aufhalten, die sowohl Gelegenheiten als auch einen Mangel an kollektiver Kontrolle aufweisen. Das Zusammentreffen von individuellen und situativen Faktoren ist demnach die zentrale Bedingung für das Entstehen von Delinquenz.

Die Untersuchung der individuellen Unterschiede kann weiteren Aufschluss über die Wirkungspfade geben, die den Einfluss der strukturellen Kontextbedingungen auf das abweichende Verhalten vermitteln, und bietet Ansatzpunkte für die Gestaltung von Präventionsund Interventionskonzepten. Im Folgenden sollen einige wichtige Dimensionen, die dabei eine Rolle spielen, kurz angesprochen werden.

Familiäre Faktoren, insbesondere die Eltern-Kind-Beziehung und das elterliche Erziehungsverhalten haben unbestritten eine zentrale Bedeutung für die Genese von Jugenddelinquenz. Eine Reihe von Studien deuten darauf hin, dass ein positives Familienklima und Erziehungsverhalten als Schutz und ,Puffer' gegen ungünstige sozialräumliche Kontexteinflüsse wirken können (Antunes/Lobo et al. 2014; Hay et al. 2007; Janssen et al. 2017). Daraus folgt umgekehrt, dass die Existenz familiärer Risikofaktoren unter ungünstigen Kontextbedingungen zu einer Verschärfung der Delinquenz führen kann. Jugendliche aus defizitären Familien in benachteiligten Wohngebieten sind demnach als besondere Risikogruppe anzusehen. Qualitative Studien haben gezeigt, dass viele Eltern große Anstrengungen unternehmen, ihre Kinder von den Gefahren der von Gewalt geprägten Armenghettos in den amerikanischen Großstädten fernzuhalten (Furstenberg et al. 1999; Molnar et al. 2005).

Auswertungen von detaillierten Zeitbudget-Bögen zeigen, dass delinquentes Verhalten dann am wahrscheinlichsten ist, wenn Jugendliche sich unorganisiert mit mehreren Freunden ohne Aufsicht von Erwachsenen in öffentlichen Räumen treffen (Hoeben et al. 2016; Wikström et al. 2012). Alkohol wirkt in diesen Situationen noch als ein Verstärker (Bernasco et al. 2013). Gemeinsame unbeaufsichtigte Freizeit lässt die Wahrscheinlichkeit delinquenter Handlungen in 
benachteiligten Wohngebieten eher ansteigen als in anderen Wohngebieten (Bernburg/Thorlindson 2007; Maimon/Browning 2010).

Aber nicht alle Jugendlichen in benachteiligten Wohngebieten oder Schulen sind selbst delinquent oder haben delinquente Freunde. Vielmehr besteht bei den Gleichaltrigenbeziehungen trotz struktureller Beschränkungen stets eine Wahlmöglichkeit, die von den Jugendlichen auch genutzt wird. In der Kölner/Freiburger Studie zeigte sich, dass der räumlichen Ausrichtung der Freundeskreise und des Freizeitverhaltens eine ganz entscheidende Bedeutung für die Frage zukommt, ob Jugendliche sich von den sozialräumlichen Bedingungen ihres Wohnquartiers beeinflussen lassen oder nicht (Oberwittler 2004c).

Eines der unerwarteten, aber mittlerweile häufig bestätigten Ergebnisse der europäischen und U.S.- amerikanischen Forschung ist, dass Mädchen stärker auf sozialräumliche Kontexteinflüsse reagieren als Jungen. Dies zeigte sich nicht nur in dem bereits erwähnten ,Moving to Opportunity'-Experiment (Burdick-Will/Ludwig 2013) und in weiteren amerikanischen Studien (Autor et al. 2015; Fagan/Wright 2012; Haynie et al. 2014), sondern auch in der Kölner/Freiburger Studie. hier fanden sich starke sozialräumliche Kontexteffekte auf die Gewaltneigung von einheimischen Mädchen, nicht aber von Jungen (Oberwittler 2003; 2007). Um zu klären, warum Mädchen in der MTO-Studie deutlicher stärker als Jungen mit einer Verringerung ihrer Gewaltdelinquenz auf den Umzug von einem extrem benachteiligten Stadtviertel in ein „,besseres“ Wohnumfeld reagierten, führten die Forscher qualitative Tiefeninterviews mit 86 der beteiligten Jugendlichen in Baltimore und Chicago durch (ClampetSundquist et al. 2011). Es zeigte sich unter anderem, dass Jungen ihren Lebensstil weniger gut als Mädchen an die veränderten Erwartungen der Nachbarschaft anpassen konnten und ihre alten Freizeitgewohnheiten - vor allem das „Herumhängen“ mit Freunden auf der Straße - tendenziell beibehielten, was zu einer erhöhten Sichtbarkeit für die lokale Polizei und zu einer Stigmatisierung durch die Bewohner der „,besseren“ Wohnquartiere führte. 


\subsection{Stadtstruktur als Ereigniskontext ${ }^{2}$}

Wenn darum geht zu erklären wo Kriminalität - gemessen an der Tatortverteilung - besonders häufig auftritt, und warum einige städtische Räume eine höhere Kriminalitätsbelastung haben als andere, konkurriert die Desorganisationstheorie mit Studien, die dem Routine Activity Approach folgen und sich bewusst von der gewohnten Perspektive auf soziale Mängellagen als Kriminalitätsursachen abgrenzen wollen. In der Bedeutung der unzureichenden Kontrolle bzw. Überwachung haben beide Ansätze eine wichtige Gemeinsamkeit, und eine Integration beider Ansätze erscheint sinnvoll und möglich (Taylor 2015).

\section{Soziale Desorganisation und kollektive Wirksamkeit - das ,systemische Modell`}

Der Desorganisationsansatz von Shaw/McKay (1969 [1942]) untersucht die kollektive Fähigkeit von Stadtvierteln, Sozialkontrolle auszuüben und dadurch Kriminalität zu verhindern. Das sogenannte ,systemische Modell' versucht das Zusammenwirken von strukturellen Merkmalen von Stadtvierteln, ihrer sozialer Organisation und dem Aufkommen von Unordnung und Kriminalität sowie auch die Rückwirkungen zwischen diesen Elementen offenzulegen (Bursik/Grasmick 1993; Kubrin/Wo 2015). Eine grundlegende Annahme des Models ist, dass die soziale Organisation von Stadtvierteln sehr stark von strukturellen Merkmalen beeinflusst wird und damit einen Mechanismus bildet, welcher die oben beschriebenen Zusammenhänge zwischen struktureller Benachteiligung und Kriminalität erklären kann. Konzentrierte Benachteiligungen verursachen nach dieser Vorstellung Kriminalität nicht direkt, sondern vermittelt über eine unzureichende kollektive Organisation der Bewohner (,,indirect effect hypothesis“, Bursik/Grasmick 1993). Dies wird in der schematischen Darstellung des Modells (Abbildung 2) dadurch angezeigt, dass der hauptsächliche Wirkungspfad von ,konzentrierter Benachteiligung“ zunächst zur ,,sozialen Organisation“ und von dort weiter zu „Kriminalität“ geht. Allerdings dauerte es bis in die 1990er Jahre, bis die Existenz dieser vermittelnden sozialen Prozesse mit Hilfe von Befragungsdaten empirisch bestätigt wurde (Bellair 1997), und auch bei Berücksichtigung der sozialen Organisation bleibt häufig ein direkter Einfluss struktureller Benachteiligung auf Kriminalität bestehen (Sampson et al. 1997). Verschiedene Studien zeigen, dass sehr enge Bindungen und intensive Interaktionen zwischen den Bewohnern weniger

\footnotetext{
${ }^{2}$ Dieser Abschnitt basiert teilweise auf Oberwittler (2013).
} 
nützlich für die Kontrolle von Kriminalität sind als schwache Bindungen (Granovetter 1973), ja dass dichte Netzwerke und Interaktionen sogar kriminalitätsfördernd wirken können, wenn sie an deviante Subkulturen gekoppelt sind (Browning et al. 2004; Warner/Rountree 1997).

Abb. 2: Zusammenhänge zwischen struktureller Benachteiligung und Kriminalität

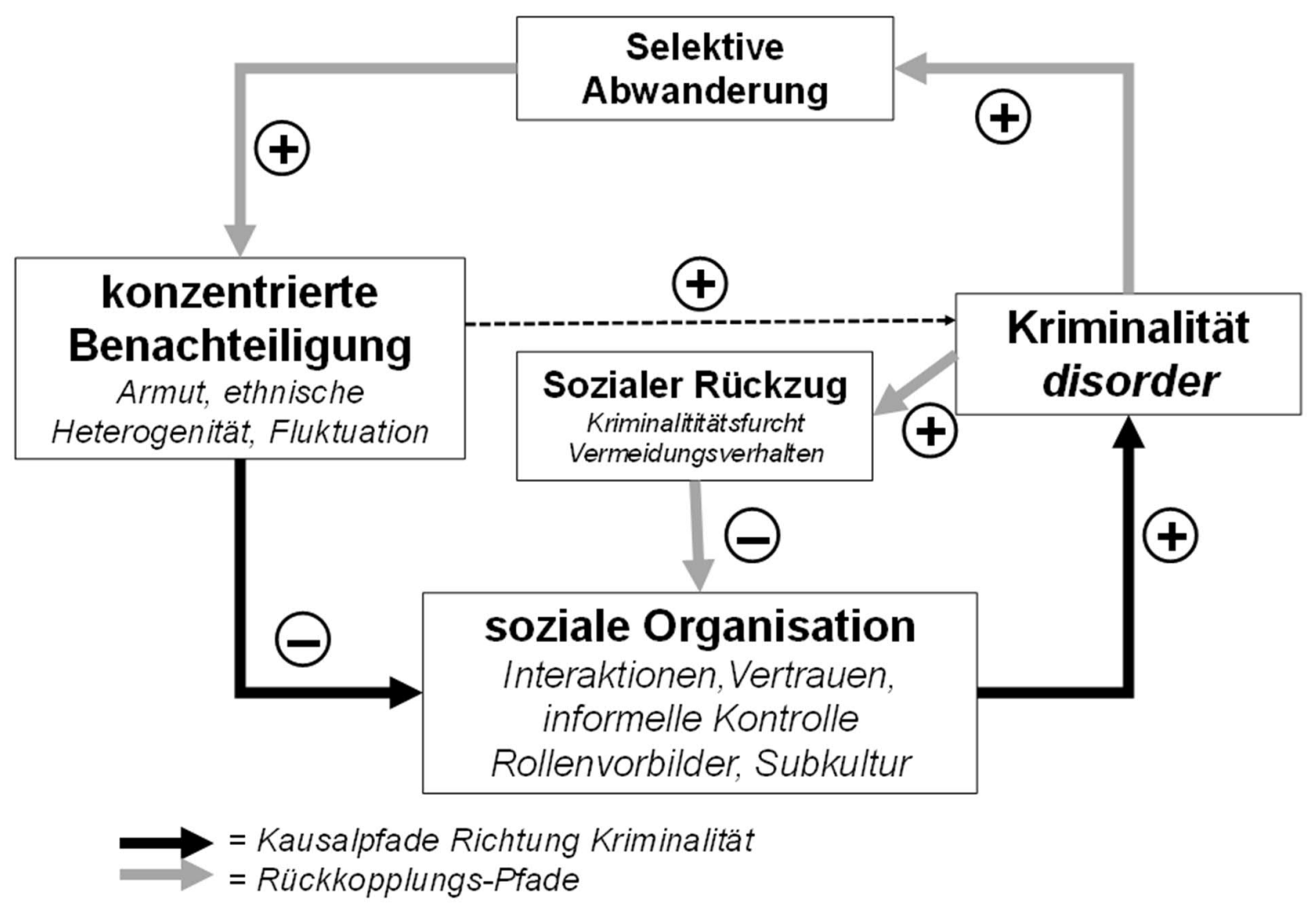

(aus: Oberwittler 2013)

Im Konzept der „kollektiven Wirksamkeit““ (collective efficacy), der von Robert Sampson (2006, 2012; Sampson et al. 1997) weiterentwickelten Version der Theorie der sozialen Desorganisation, wird daher weniger Wert auf soziale Netzwerke als auf generalisiertes 
Vertrauen und Verhaltenserwartungen im kollektiven Interesse gelegt. Die Kernidee der kollektiven Wirksamkeit ist, dass sich Bewohner dann erfolgreich für das gemeinsame Wohl der Nachbarschaft einsetzen - konkret indem sie bei Fehlverhalten von Kindern und Jugendlichen einschreiten -, wenn eine Basis gegenseitigen Vertrauens und geteilter Normen vorhanden ist. Erkenntnisse aus ethnographischen Studien (Carr 2003) trugen dazu bei, dass im Konzept der kollektiven Wirksamkeit auch das Einschalten der Polizei unter den Begriff der informellen Sozialkontrolle subsumiert wird, solange die Initiative dazu von den Bewohnern ausgeht und es sich nicht um eine pro-aktive Polizeistrategie handelt (Sampson 2006, S. 40). Ein vertrauensvolles Verhältnis zwischen den Bewohnern und Vertrauen in die Polizei werden heute nicht als widersprüchlich gesehen (Kochel 2016).

Das Konzept der kollektiven Wirksamkeit ist in der kriminalsoziologischen Forschung enorm erfolgreich und wurde in vielen Studien angewendet, so z.B. in Schweden (Sampson/Wikström 2008), Großbritannien (Wikström et al. 2012) und Australien (Mazerolle et al. 2010). Aber nicht immer bestätigten sich signifikante Effekte auf Gewaltkriminalität (Bruinsma et al. 2013), so auch in einigen der seltenen Längsschnittstudien, die besser zur Identifizierung kausaler Wirkungen geeignet sind (Hipp/Wickes 2016). Wiederholt zeigte sich der paradoxe Befund, dass zwar die hypothetischen Erwartungen an die Handlungsbereitschaft der Nachbarn mit zunehmenden Problemlagen im Wohngebiet sinken, jedoch gleichzeitig die tatsächlich praktizierte informelle Sozialkontrolle zunimmt (Lüdemann 2006b; Steenbeek/Hipp 2011; Wickes et al. 2016; vgl. dazu Sampson 2006, S. 40).

Rückwirkungen von Kriminalität und Unordnung - Broken Windows Theory

Ein sehr wichtiger Teil des systemischen Modells befasst sich mit den Rückwirkungen von Kriminalität sowie verschiedener Formen der Unordnung auf die soziale Organisation von Stadtvierteln. Persönliche Erfahrungen, Beobachtungen oder Berichte von Kriminalität und Unordnung können Unsicherheitswahrnehmungen fördern, Vertrauen zerstören, zu sozialem 
Rückzug führen und so die kollektive Wirksamkeit von Stadtvierteln vermindern. Diese Rückwirkungen sind in Abbildung 2 als graue Pfeile dargestellt.

Es waren vor allem zwei Erklärungsansätze, die die Auswirkungen urbaner Unordnung und Unsicherheitswahrnehmungen auf Wohnquartiere in den Mittelpunkt gerückt und eine Flut von weiteren Studien ausgelöst haben: Wesley Skogan argumentierte in seinem Buch Disorder and Decline (1990, vgl. Skogan 2015), dass benachteiligte Wohnquartiere der amerikanischen Großstädte durch Kriminalität und Unordnung in eine Abwärtsspirale des Verfalls hineingezogen werden, und Wilson und Kelling postulierten in ihrem berühmt gewordenen Aufsatz Broken Windows (1982) einen Mechanismus, demzufolge alltägliche Phänomene physischer und sozialer Unordnung wie Müll, zerbrochene Fensterscheiben oder lärmende Jugendliche als sichtbarer Ausdruck einer mangelnden Sozialkontrolle im öffentlichen Raum die Bewohner weiter verunsichern und potenzielle Täter zur Kriminalität animieren. In einer Längsschnittstudie mit Daten des British Crime Surveys fanden Markowitz et al. (2001) Hinweise auf einen solchen negativen Feedback-Kreislauf zwischen Unordnung, Angst und Vertrauen: „Cohesion decreases disorder, which increases fear, which decreases cohesion” (Markowitz et al. 2001, S. 310). Weitere Studien haben die Annahme negativer Rückwirkungen von Kriminalität und Unordnung bestätigt, teils nur in Queerschnittsstudien (Gault/Silver 2008; Garcia et al. 2007; Häfele 2013), teils auch in Längssschnittstudien (Brunton-Smith 2011; Steenbeck/Hipp 2011).

Es ist jedoch umstritten, ob Unordnung neben Kriminalität eine eigenständige kausale Rolle im systemischen Modell spielt (Gau/Pratt 2008; Raudenbush/Sampson 1999; Sampson 2009). Eine methodisch besonders anspruchsvolle Längsschnittstudie in Baltimore fand keinen signifikanten Effekt von Unordnung auf eine spätere Zunahme von Kriminalitätsfurcht oder auf eine abnehmende Zufriedenheit mit dem Wohnquartier (Robinson et al. 2003). Ralph Taylor und seine Kollegen argumentieren, dass diese starke Korrelation zwischen der Wahrnehmung von Unordnung und Kriminalitätsfurcht Ausdruck einer Überschneidung der Bedeutungsinhalte der Fragen ist und deswegen erheblich überschätzt wird: Befragte, die sehr ängstlich sind, nehmen auch mehr Unordnung war (Covington/Taylor 1991; Link et al. 2017). Die subjektiven Wahrnehmungen des städtischen Kontextes werden von unterschiedlichen Empfindsamkeiten beeinflusst und drücken auch diffuse, allgemeinere gesellschaftliche Sorgen aus (Farrall et al. 2009; Hirtenlehner/Farrall 2013). Aus diesem Grunde wurden in einigen Studien systematische Beobachtungen physischer und sozialer Zeichen der Unordnung durch trainierte Mitarbeiter 
durchgeführt (Häfele 2013; Sampson/Raudenbush 1999). Diese objektiv gemessene Unordnung hat deutlich geringere Effekte auf Unsicherheitswahrnehmungen (Hinkle/Yang 2014; Häfele 2013; Perkins/Taylor 1996), ebenso wie auch die tatsächliche Kriminalitätsbelastung (Lüdemann 2006a). Die subjektiven Wahrnehmungen von Unordnung werden hingegen von der ethnischen Zusammensetzung der Wohnquartiere mitbestimmt: Relativ zu den systematischen Beobachtungen nehmen Bewohner in Stadtvierteln mit hohem Migrantenanteil mehr Unordnung wahr, und zwar unabhängig von ihrem eigenen sozialen und ethischen Status (Janssen et al. eingereicht; Sampson/Raudenbush 2004). Die Gültigkeit des

Broken Windows Ansatzes bleibt daher umstritten, und erst recht was den Erfolg der darauf basierenden Polizeistrategien betrifft (Harcourt/Ludwig 2007).

\section{Urbane Gelegenheitsstrukturen und ,Hot Spots`}

Räumliche Kriminalitätsschwerpunkte in Großstädten werden stark von Gelegenheiten bestimmt, denn Kriminalität folgt den Alltagsroutinen der Menschen mit typischen geographischen Mustern. Mit der digitalen Verfügbarkeit präziser Tatortdaten und mithilfe von Crime Mapping wurde sehr deutlich, wie stark insbesondere Gewalt in den Innenstädten auf kleine ,Hot Spots ‘ konzentriert und mit dem räumlichen (und zeitgebundenen) Nutzungsverhalten verknüpft ist (Sherman/Gartin et al. 1989; Weisburd 2015). Aus diesen Beobachtungen hat sich eine eigene, ,Environmental Criminology ' genannte Forschungsrichtung gebildet, die sich bewusst von der ,traditionellen`, auf die Täter fokussierten Kriminologie absetzen möchte (Andresen 2014). Ihre theoretische Basis liefert der Routine Activity Approach (Cohen/Felson 1979), für den drei Bedingungen in Raum und Zeit zusammentreffen müssen, damit Kriminalität entsteht: motivierte Täter, attraktive Ziele und ein Mangel an effektiver Überwachung. Die Environmental Criminology interessiert sich vorrangig für das „Wo, Wann und Wie der Kriminalität“, aber nicht für das „Warum“ (Townsley 2017, S. 101, Übersetzung d. Verf.) Die Existenz motivierter 
Täter wird zwar vorausgesetzt, im Mittelpunkt stehen jedoch die als eigenständige Ursachen interpretierten Effekte der Gelegenheitsstrukturen. Das Menschenbild dieses Ansatzes ist der des ,Reasoning Criminal`, der vernünftige und die Chancen und Risiken einer Straftat abwägende Täter (Cornish/Clarke 1986), dessen Verhalten in einigen Studien bereits mit, Agent Based Modeling'-Programmen simuliert wird (Birks et al. 2012). Dem entspricht eine pragmatische Orientierung auf situative, technische und städteplanerische Maßnahmen der Kriminalprävention (Clarke 1995).

Empirische Studien der Environmental Criminology verwenden häufig nicht Stadtviertel, sondern Straßenabschnitte als kleinste Einheiten, um die extreme räumliche Konzentration von Tatorten abzubilden (Sherman et al. 1989). Hauptstraßen, Haltestellen und eine gemischte Landnutzung mit Firmen, Geschäften, Restaurants und Bars sind Elemente von Gelegenheitsstrukturen, die diese Konzentration erklären können, auch unter Berücksichtigung von sozialstrukturellen Problemlagen (Weisburd et al. 2012). Je feiner die räumliche Auflösung der Analyse und je kleiner damit die räumliche Distanz zu solchen geographischen Merkmalen, desto stärker sind die Zusammenhänge mit Kriminalität, während die Effekte sozialstruktureller Benachteiligung sich bei umgekehrt in größeren räumlichen Einheiten deutlicher zeigen (Groff/Lockwood 2014). Wenn innenstädtische Geschäfts- und Vergnügungsviertel eine besonders hohe Kriminalitätsbelastung aufweisen, so muss dies angesichts der dort ebenfalls sehr großen Aufenthaltsbevölkerung nicht zwangsläufig ein erhöhtes individuelles Kriminalitätsrisiko bedeuten: Da die Größe der Aufenthaltsbevölkerung kaum messbar ist, stehen die Einflussstärken der Indikatoren der Gelegenheitsstrukturen in vielen Studien unter einem Vorbehalt (Andresen/Jenion 2010; Oberwittler 2004b).

Für Wohnungseinbrüche sind u.a. Grundstücksgestaltung, technische Sicherungen, Erreichbarkeit im Straßennetz und Nähe zu Wohnsitzen potenzieller Täter relevant (Davies/Johnson 2015; Montoya et al. 2016). Die Identifizierung von lokalisierten Serien von Wohnungseinbrüchen in kurzem zeitlichen Abstand (,Near Repeats') hat zur Entwicklung von Crime Mapping-basierten Vorhersageprogrammen geführt, die zur Prävention zukünftiger Taten beitragen sollen (Gerstner 2017; Perry et al. 2013). 
Die Wirkung der baulichen Struktur von Wohngebieten auf Kriminalität, die in Oscar Newmans architektonischem Konzept des ,Defensible Space` und darauf aufbauenden Ansätzen der „Crime Prevention Through Environmental Design“ (CPTED) in den Mittelpunkt gerückt wurde, ist jedoch empirisch schlecht abgesichert und umstritten (Reynald/Elffers 2009). Für die hohe Kriminalitätsbelastung vieler Großwohnsiedlungen ist das bauliche Design vermutlich in erster Linie indirekt, durch die ungünstige soziodemographische Zusammensetzung der Bewohner, verantwortlich (MacDonald 2015).

Eine Integration von Täter- und Situations-orientierten Ansätzen in der Analyse urbaner Kriminalität ist nicht nur wünschenswert, sondern auch in verschiedenen Theorien wie dem Routine Activity Approach und der Situational Action Theory bereits angelegt. Nimmt man deren Grundannahme ernst, so entsteht Kriminalität vor allem dort, wo motivierte Täter und geeignete Gelegenheiten gemeinsam auftreten (Weisburd et al. 2012: S. 43, vgl.

Gerstner/Oberwittler 2011; Smith et al. 2000). Die Verbindung von Personen- und Raumperspektive sowie von Individual- und räumlichen Kontextdaten ist bislang in der Situational Action Theory und den darauf aufbauenden Studien am konsequentesten umgesetzt worden (Wikström et al. 2012, S. 315, 352). Diese Ergebnisse unterstreichen, dass ein Verständnis städtischer Kriminalitätsphänomene ohne die Berücksichtigung der Täter und der ,klassischen` Fragen nach ihren Handlungsdispositionen nicht sinnvoll ist. 


\section{Literaturverzeichnis}

Andresen, M.A. (2014): Environmental criminology. Evolution, theory and practice. London: Routledge.

Andresen, M.A./Jenion, G.W. (2010): Ambient populations and the calculation of crime rates and risk. In: Security Journal 23, S. 114-133.

Antunes, M.J.L./Ahlin, E.M. (2014): Family management and youth violence: are parents or community more salient? In: Journal of Community Psychology 42(3), S. 316-337.

Autor, D./Figlio, D./Karbownik, K./Roth, J./ Wasserman, M. (2015). Family Disadvantage and the Gender Gap in Behavioral and Educational Outcomes (Institute for Policy Research, Northwestern University, Working Paper 15-16).

Baumer, E.P./Arnio, A.N. (2015): Macro-Level Theory. In: Piquero, A. (Hg.), The Handbook of Criminological Theory. John Wiley \& Sons, Inc, S. 445-474.

Beier, H. (2014): Peer effects in offending behaviour across contexts: Disentangling selection, opportunity and learning processes. In: European Journal of Criminology 11(1), S. 73-90.

Belina, B./Wehrheim, J. (2011): „Gefahrengebiete" - Durch die Abstraktion vom Sozialen zur Reproduktion gesellschaftlicher Strukturen. In: Oberwittler, D./Behr, R. (Hg.), Polizei und Polizieren in multiethnischen Gesellschaften. Freiburg: Centaurus, S. 207-230.

Bellair, P.E. (1997): Social interaction and community crime: Examining the importance of neighbor networks. In: Criminology 35, S. 677-703.

Bellair, P.E./McNulty, T.L. (2005): Beyond the Bell Curve: Community Disadvantage and the Explanation of Black-White Differences in Adolescent Violence. In: Criminology 43(4), S. 1135-1167.

Bernasco, W./Ruiter, S./Bruinsma, G.J./Pauwels, L.J./Weerman, F.M. (2013): Situational causes of offending: a fixed-effects analysis of space-time budget data. In: Criminology 51(4), S. 895-926.

Bernburg, J.G./Thorlindsson, T. (2007): Community structure and adolescent delinquency in Iceland: A contextual analysis. In: Criminology 45(2), S. 415-444. 
Bingenheimer, J.B./Brennan, R.T./Earls, F.J. (2005): Firearm Violence Exposure and Serious Violent Behavior. In: Science 308, S. 1323-1326.

Birks, D./Townsley, M./Stewart, A. (2012): Generative Explanations of Crime: Using Simulation to Test Criminological Theory. In: Criminology 50(1), S. 221-254.

Boers, K./Reinecke, J./Mariotti, L./Seddig, D. (2010): Explaining the Development of Adolescent Violent Delinquency. In: European Journal of Criminology 7(6), S. 499-520.

Bottoms, A.E. (2012): Developing socio-spatial criminology. In: Maguire, M./Morgan, R./Reiner, R. (Hg.), The Oxford Handbook of Criminology (5.A.). Oxford: Oxford University Press, S. 450-488

Brännström, L./Rojas, Y. (2012): Rethinking the Long-Term Consequences of Growing Up in a Disadvantaged Neighbourhood: Lessons from Sweden. In: Housing Studies 27(6), S. 729747.

Brantingham, P.L./Brantingham, P.J. (1995): Criminality of place. Crime generators and crime attractors. In: European Journal on Criminal Policy and Research 3(3), S. 5-26.

Brantingham, P.J./Brantingham, P.L. (2008): Crime pattern theory. In: Wortley, R./Mazerolle, L. (Hg.), Environmental Criminology and Crime Analysis. Cullompton: Willan, S. 78-93.

Browning, C.R./Feinberg, S.L./Dietz, R.D. (2004): The Paradox of Social Organization: Networks, Collective Efficacy, and Violent Crime in Neighborhoods. In: Social Forces 83(2), S. 503-534.

Brunton-Smith, I. (2011): Untangling the relationship between fear of crime and perceptions of disorder. Evidence from a Longitudinal Study of Young People in England and Wales. In: British Journal of Criminology 51, S. 885-899.

Burdick-Will, J./Ludwig, J. (2013): Neighborhood Effects on Crime. Evidence from America's Moving to Opportunity Experiment. In: Oberwittler, D./Rabold, S./Baier, D. (Hg.), 
Städtische Armutsquartiere - Kriminelle Lebenswelten?. Wiesbaden: Springer VS, S. 97116.

Bursik, R.J./Grasmick, H.G. (1993): Neighborhoods and crime: the dimensions of effective community control. New York: Lexington Books.

Busch, H./Werkentin, F. (1992): Die soziale Produktion polizeilich registrierter Gewaltindizien. In: Brusten, M. (Hg.), Polizei-Politik. Weinheim: Juventa, S. 69-83.

Carr, P. (2003): The New Parochialism: The Implications of the Beltway Case for Arguments Concerning Informal Social Control. In: American Journal of Sociology 108(6), S. 12491291.

Chamberlain, A.W./Hipp, J.R. (2015): It's all relative: Concentrated disadvantage within and across neighborhoods and communities, and the consequences for neighborhood crime. In: Journal of Criminal Justice 43(6), S. 431-443.

Chang, L.-Y./Wang, M.-Y./Tsai, P.-S. (2016): Neighborhood disadvantage and physical aggression in children and adolescents: A systematic review and meta-analysis of multilevel studies. In: Aggressive Behavior 42, S. 441-454.

Clampet-Lundquist, S./Edin, K./Kling, J.R./Duncan, G.J. (2011): Moving Teenagers Out of High-Risk Neighborhoods: How Girls Fare Better than Boys. In: American Journal of Sociology 116(4), S. 1154-1189.

Clarke, R.V. (1995): Situational crime prevention. In: Tonry, M. (Hg.), Building a Safer Society: Strategic Approaches to Crime (Crime and Justice, vol. 19). Chicago: Chicago University Press, S. 91-150.

Cohen, L.E./Felson, M. (1979): Social Change and Crime Rate Trends: A Routine Activity Approach. In: American Sociological Review 44(4), S. 588-608.

Coleman, J.S. (1990): Foundations of Social Theory. Cambridge, Mass.: Harvard University Press.

Cornish, D.A./Clarke, R.V. (Hg.) (1986): The Reasoning criminal: rational choice perspectives on offending. New York: Springer.

Covington, J./Taylor, R.B. (1991): Fear of crime in urban residential neighborhoods: 
Implications of between and within-neighborhood sources for current models. In: The Sociological Quarterly (32), S. 231-249.

Crane, J. (1991): The epidemic theory of ghettos and neighborhood effects on dropping out and teenage childbearing. In: American Journal of Sociology 96, S. 1226-1259.

Crutchfield, R.D. (2015): From Slavery to Social Class to Disadvantage: An Intellectual History of the Use of Class to Explain Racial Differences in Criminal Involvement. In: Crime and Justice 44(1), S. 1-47.

Damm, A.P./Dustmann, C. (2014): Does Growing Up in a High Crime Neighborhood Affect Youth Criminal Behavior? In: American Economic Review 104(6), S. 1806-1832.

Davies, T./Johnson, S.D. (2015): Examining the Relationship Between Road Structure and Burglary Risk Via Quantitative Network Analysis. In: Journal of Quantitative Criminology 31(3), S. 481-507.

De Coster, S./Heimer, K.;/Wittrock, S.M. (2006): Neighborhood disadvantage, social capital, street context, and youth crime. In: Sociological Quarterly 47(4), S. 723-753.

Eisner, M. (1997): Das Ende der zivilisierten Stadt? Die Auswirkungen von Modernisierung und urbaner Krise auf Gewaltdelinquenz. Frankfurt am Main: Campus.

Elliott, D.S./Menard, S./Rankin, B.H./Wilson, W.J./Huizinga, D. (2006): Good Kids from Bad Neighborhoods. Successful Development in Social Context. Cambridge: Cambridge University Press.

Engels, F. (1845): Die Lage der arbeitenden Klasse in England. Leipzig: Wigand.

Estrada, F./Nilsson, A. (2008): Segregation and Victimization. Neighbourhood Resources, Individual Risk Factors and Exposure to Property Crime. In: European Journal of Criminology 5(2), S. 193-216.

Fagan, A.A./Wright, E.M. (2012): The Effects of Neighborhood Context on Youth Violence and Delinquency. Does Gender Matter? In: Youth Violence and Juvenile Justice 10(1), S. 64-82. 
Farrall, S./Jackson, J./Gray, E. (2009): Social order and the fear of crime in contemporary times. Oxford: Oxford University Press.

Farrell, G./Tilley, N./Tseloni, A. (2014): Why the Crime Drop? In: Crime and Justice 43(1), S. 421-490.

Frehsee, D. (1979): Strukturbedingungen urbaner Kriminalität: Eine Kriminalgeographie der Stadt Kiel unter besonderer Berücksichtigung der Jugendkriminalität. Göttingen: Otto Schwarz.

Frevel, B. (Hg.) (2012): Handlungsfelder lokaler Sicherheitspolitik. Netzwerke, Politikgestaltung und Perspektiven. Frankfurt: Verlag für Polizeiwissenschaften.

Furstenberg, F.F.J./Cook, T.D./Eccles, J./Elder, G.H.J./Sameroff, A. (1999): Managing to Make It. Urban Families and Adolescent Success. Chicago: University of Chicago Press.

Garcia, R.M./Taylor, R.B./Lawton, B.A. (2007): Impacts of Violent Crime and Neighborhood Structure on Trusting Your Neighbors. In: Justice Quarterly 24(4), S. 679-704.

Gau, J.M./Pratt, T.C. (2008): Broken windows or window dressing? Citizens (in)ability to tell the difference between disorder and crime. In: Criminology \& Public Policy 7(2), S. 163 194.

Gault, M./Silver, E. (2008): Spuriousness or mediation? Broken windows according to Sampson and Raudenbush (1999). In: Journal of Criminal Justice 36, S. 240-243.

Gerstner, D. (2017): Predictive Policing als Instrument zur Prävention von Wohnungseinbruchdiebstahl. Evaluationsergebnisse zum Baden-Württembergischen Pilotprojekt P4 (Forschung Aktuell / 49). Freiburg: Max-Planck-Institut für ausländisches und internationales Strafrecht.

Gerstner, D./Oberwittler, D. (2015): Wer kennt wen und was geht ab? Ein netzwerkanalytischer Blick auf die Rolle delinquenter Peers im Rahmen der ,Situational Action Theory'. In: Monatsschrift für Kriminologie und Strafrechtsreform 2015(3), S. 204-226.

Granovetter, M. (1973): The Strength of Weak Ties. In: American Journal of Sociology 78(6), S. 1360-1380.

Groff, E.R./Lockwood, B. (2014): Criminogenic Facilities and Crime across Street Segments in 
Philadelphia: Uncovering Evidence about the Spatial Extent of Facility Influence. In: Journal of Research in Crime and Delinquency 51(3), S. 277-314.

Häfele, J. (2013): Die Stadt, das Fremde und die Furcht vor Kriminalität. Eine Mehrebenenanalyse zum Einfluss von urbanen Disorder-Phänomenen auf personale Kriminalitätseinstellungen. Wiesbaden: Springer VS.

Harcourt, B.E./Ludwig, J. (2007): Reefer Madness: Broken Windows Policing And Misdemeanor Marijuana Arrests In New York City, 1989-2000. In: Criminology \& Public Policy 6(1), S. 165-182.

Harding, D.J. (2009): Violence, Older Peers, and the Socialization of Adolescent Boys in Disadvantaged Neighborhoods. In: American Sociological Review 74, S. 445-464.

Hay, C./Fortson, E.N./Hollist, D.R./Altheimer, I./Schaible, L.M. (2007): Compounded Risk: The Implications for Delinquency of Coming from a Poor Family that Lives in a Poor Community. In: Journal of Youth and Adolescence 36, S. 593-605.

Haynie, D.L./Kreager, D.A. (2013): Peer networks and crime. In: Cullen, F.T./Wilcox, P. (Hg.), The Oxford handbook of criminological theory. New York/Oxford: Oxford University Press, S. 257-273.

Haynie, D.L./Doogan, N.J./Soller, B. (2014): Gender, Friendship Networks, and Delinquency: A Dynamic Network Approach. In: Criminology 52(4), S. 688-722.

Hermann, D./Laue, C. (2004): Vom „Broken-Windows-Ansatz" zu einer lebensstilorientierten ökologischen Kriminalitätstheorie. In: Soziale Probleme 14(2), S. 107-137.

Hinkle, J.C./Yang, S.-M. (2014): A New Look into Broken Windows: What Shapes Individuals' Perceptions of Social Disorder? In: Journal of Criminal Justice 42(1), S. 26-35.

Hipp, J.R. (2010): A Dynamic View of Neighborhoods: The Reciprocal Relationship between Crime and Neighborhood Structural Characteristics. In: Social Problems 57(2), S. 205-230. Hipp, J.R./Wickes, R. (2016): Violence in Urban Neighborhoods: A Longitudinal Study of Collective Efficacy and Violent Crime. In: Journal of Quantitative Criminology, S. 1-26. Hipp, J.R./Steenbeek, W. (2016): Types of Crime and Types of Mechanisms: What Are the Consequences for Neighborhoods Over Time? In: Crime \& Delinquency 62(9), S. 1203- 
1234.

Hirtenlehner, H./Farrall, S. (2013): Anxieties About Modernization, Concerns About Community, and Fear of Crime: Testing Two Related Models. In: International Criminal Justice Review 23(1), S. 5-24.

Hoeben, E.M./Meldrum, R.C./Walker, D./Young, J.T. (2016): The role of peer delinquency and unstructured socializing in explaining delinquency and substance use: A state-of-the-art review. In: Journal of Criminal Justice 47, S. 108-122.

Hurrelmann, K. (2006): Einführung in die Sozialisationstheorie (9. A.). Weinheim: Beltz. Janssen, H./Oberwittler, D./Gerstner, D. Perceptions of Disorder and the Concentration of Visible Minorities: The Moderating Role of Interethnic Contact and Xenophobic Attitudes, eingereicht.

Janssen, H.J./Weerman, F.M./Eichelsheim, V.I. (2017): Parenting as a Protective Factor against Criminogenic Settings? Interaction Effects between Three Aspects of Parenting and Unstructured Socializing in Disordered Areas. In: Journal of Research in Crime and Delinquency 54(2), S. 181-207.

Johnson, S.D./Bowers, K.J. (2004): The Burglary as Clue to the Future. In: European Journal of Criminology 1(2), S. 237-255.

Kirk, D.S. (2008): The Neighborhood Context of Racial and Ethnic Disparities in Arrest. In: Demography 45(1), S. 55-77.

Klein, M.W./Weerman, F.M./Thornberry, T.P. (2006): Street Gang Violence in Europe. In: European Journal of Criminology 3(4), S. 413-437.

Kochel, T.R. (2016): Police legitimacy and resident cooperation in crime hotspots: effects of victimisation risk and collective efficacy. In: Policing and Society, S. 1-20. 
Lauritsen, J.L. (2001): The social Ecology of Violent Victimization: Individual and Contextual Effects in the NCVS. In: Journal of Quantitative Criminology 17(1), S. 3-32.

Link, N.W./Kelly, J.M./Pitts, J.R./Waltman-Spreha, K./Taylor, R.B. (2017): Reversing Broken Windows: Evidence of Lagged, Multilevel Impacts of Risk Perceptions on Perceptions of Incivility. In: Crime \& Delinquency 63(6), S. 659-682.

Ludwig, J./Liebman, J.B./Kling, J.R./Duncan, G.J./Katz, L.F./Kessler, R.C./Sanbonmatsu, L. (2008): What Can We Learn about Neighborhood Effects from the Moving to Opportunity Experiment? In: American Journal of Sociology 114(1), S. 144-188.

Lüdemann, C. (2006): Soziales Kapital und soziale Kontrolle. Zu den Determinanten sozialer Kontrolle in Nachbarschaften. In: Kriminalistik 60(3), S. 177-182.

Lüdemann, C. (2006): Kriminalitätsfurcht im urbanen Raum. Eine Mehrebenenanalyse zu individuellen und sozialräumlichen Determinanten von Kriminalitätsfurcht im städtischen Raum. In: Kölner Zeitschrift für Soziologie und Sozialpsychologie 58, S. 285-306.

MacDonald, J. (2015): Community Design and Crime: The Impact of Housing and the Built Environment. In: Crime and Justice 44(1), S. 333-383.

Maimon, D./Browning, C.R. (2010): Unstructured socializing, collective efficacy, and violent behavior among urban youth. In: Criminology 48(2), S. 443-474.

Markowitz, F.E./Bellair, P.E./Liska, A.E./Liu, J. (2001): Extending Social Disorganization Theory: Modeling the Relationships between Cohesion, Disorder, and Fear. In: Criminology 39(2), S. 293-319.

Mayhew, H. (1861): London labour and the London poor: the condition and earnings of those that will work, those that cannot work, and those that will not work. London: Griffin.

Mazerolle, L.G./Wickes, R./McBroom, J. (2010): Community Variations in Violence: The Role of Social Ties and Collective Efficacy in Comparative Context. In: Journal of Research in Crime and Delinquency 47(1), S. 3-30.

Molnar, B.E./Roberts, A.L./Browne, A./Gardener, H./Buka, S.L. (2005): What girls need: recommendations for preventing violence among urban girls in the US. In: Social Science \& Medicine 60, S. 2191-2204. 
Montoya, L./Junger, M./Ongena, Y. (2016): The Relation Between Residential Property and Its Surroundings and Day- and Night-Time Residential Burglary. In: Environment and Behavior 48(4), S. 515-549.

Morenoff, J.D./Sampson, R.J. (1997): Violent Crime and the Spatial Dynamics of Neighborhood Transition: Chicago, 1970-1990. In: Social Forces 76 (1), S. 31-64.

Müller, P.F./Mueller, M. (2011): Chicago am Rhein. Geschichten aus dem Kölschen Milieu. Köln: Kiepenheuer \& Witsch.

Nieuwenhuis, J./Hooimeijer, P. (2016): The association between neighbourhoods and educational achievement, a systematic review and meta-analysis. In: Journal of Housing and the Built Environment 31(2), S. 321-347.

Oberwittler, D. (2001): Täter, Opfer, Tatorte. Zu den innerstädtischen Verteilungsmustern der registrierten Gewaltkriminalität und ihren sozialen Korrelaten am Beispiel Kölns. In: Jehle, J.-M. (Hg.), Raum und Kriminalität: Sicherheit in der Stadt, Migrationsprobleme. Mönchengladbach: Forum Verlag Godesberg, S. 121-146

Oberwittler, D. (2003): Geschlecht, Ethnizität und sozialräumliche Benachteiligung überraschende Interaktionen bei sozialen Bedingungsfaktoren von Gewalt und schwerer Eigentumsdelinquenz von Jugendlichen. In: Lamnek, S./Boatca, M. (Hg.), Geschlecht Gewalt - Gesellschaft. Opladen: Leske + Budrich, S. 269-294

Oberwittler, D. (2004a): A Multilevel Analysis of Neighbourhood Contextual Effects on Serious Juvenile Offending. The Role of Subcultural Values and Social Disorganization. In: European Journal of Criminology 1(2), S. 201-235.

Oberwittler, D. (2004b). Re-Balancing Routine Activity and Social Disorganization Theories in the Explanation of Urban Violence: A New Approach to the Analysis of Spatial Crime Patterns Based on Popula- 
tion at Risk (working paper of the project "Social Problems and Juvenile Delinquency in Ecological Perspective No. 10).

Oberwittler, D. (2004c): Stadtstruktur, Freundeskreise und Delinquenz. Eine Mehrebenenanalyse zu sozialökologischen Kontexteffekten auf schwere Jugenddelinquenz. In: Oberwittler, D./Karstedt, S. (Hg.), Soziologie der Kriminalität. Wiesbaden: VS Verlag für Sozialwissenschaften, S. 135-170.

Oberwittler, D. (2007): The Effects of Neighbourhood Poverty on Adolescent Problem Behaviours: A Multi-level Analysis Differentiated by Gender and Ethnicity. In: Housing Studies 22(5), S. 781-803.

Oberwittler, D. (2008): Armut macht Angst. Ansätze einer sozialökologischen Interpretation der Kriminalitätsfurcht. In: Groenemeyer, A./Wieseler, S. (Hg.), Soziologie sozialer Probleme und sozialer Kontrolle. Realitäten, Repräsentationen und Politik. Festschrift für Günter Albrecht. Wiesbaden: VS Verlag für Sozialwissenschaften, S. 215-230.

Oberwittler, D. (2012): Delinquenz und Kriminalität als soziales Problem. In: Albrecht, G./Groenemeyer, A. (Hg.), Handbuch Soziale Probleme (2.A.). Wiesbaden: Springer VS, S. $772-860$.

Oberwittler, D. (2013): Wohnquartiere und Kriminalität - Überblick über die Forschung zu den sozialräumlichen Dimensionen urbaner Kriminalität. In: Oberwittler, D./Rabold, S./Baier, D. (Hg.), Städtische Armutsquartiere - Kriminelle Lebenswelten? Studien zu sozialräumlichen Kontexteffekten auf Jugendkriminalität und Kriminalitätswahrnehmungen. Wiesbaden: Springer VS, S. 45-96.

Oberwittler, D./Gerstner, D. (2011): Kriminalgeographie Baden-Württembergs (2003-2007). Sozio-ökonomische und räumliche Determinanten der registrierten Kriminalität. Freiburg: Max-Planck-Institut für ausländisches und internationales Strafrecht.

Oberwittler, D./Gerstner, Dominik (2016): Kriminalitätsfurcht in großstädtischen Wohngebieten - Wie sozialräumliche Bedingungen die Unsicherheitswahrnehmungen beeinflussen. In: Zoche, P./Kaufmann, S./Arnold, H. (Hg.), Grenzenlose Sicherheit? Gesellschaftliche Dimensionen der Sicherheitsforschung. Berlin: Lit, S. 95-116.

Opp, K.-D. (1968): Zur Erklärung delinquenten Verhaltens von Kindern und Jugendlichen. Eine 
ökologische Analyse der Kinder- und Jugenddelinquenz in Köln und eine Kritik des kriminal-ökologischen Ansatzes. München: Deutsches Jugendinstitut.

Opp, K.-D. (2015): Modellbildung und Simulation: Einige methodologische Fragen. In: Braun,

N./Saam, N.J. (Hg.), Handbuch Modellbildung und Simulation in den Sozialwissenschaften.

Wiesbaden: Springer VS, S. 181-212.

Perkins, D.D./Taylor, R.B. (1996): Ecological Assessments of Community Disorder - Their Relationship to Fear of Crime and Theoretical Implications. In: American Journal of Community Psychology 24(1), S. 63-107.

Perry, W.L./McInnis, B./Price, C.C./Smith, S.C./Hollywood, J.S. (2013): Predictive Policing:

The Role of Crime Forecasting in Law Enforcement Operations (RAND Safety and Justice Program). Santa Monica: Rand.

Peterson, R.D./Krivo, L.J. (2010): Divergent Social Worlds: Neighborhood Crime and the Racial-Spatial Divide. New York: Russell Sage Foundation.

Pinker, S. (2011): The Better Angels of Our Nature: Why Violence Has Declined. Penguin. Pratt, T.C./Cullen, F.T. (2005): Assessing Macro-Level Predictors and Theories of Crime: A Meta-Analysis. In: Tonry, M. (Hg.), Crime and Justice. An Annual Review of Research. Vol. 32. Chicago: Chicago University Press, S. 373-450.

Raudenbush, S.W./Sampson, R.J. (1999): Ecometrics: Toward a Science of Assessing Ecological Settings, with Appliance to the Systematic Social Observation of Neighborhoods. In: Sociological Methodology 29, S. 1-41.

Reynald, D.M./Elffers, H. (2009): The Future of Newman's Defensible Space Theory: Linking Defensible Space and the Routine Activities of Place. In: European Journal of Criminology $6(1)$, S. 25-46.

Robinson, J.B./Lawton, B.A./Taylor, R.B./Perkins, D.D. (2003): Multilevel Longitudinal Impacts of Incivilities: Fear of Crime, Expected Safety, and Block Satisfaction. In: Journal of Quantitative Criminology 19(3), S. 237-274.

Roncek, D.W./ Maier, P.A. (1991): Bars, Blocks, and Crimes Revisited: Linking the Theory of Routine Activities to the Empiricism of Hot Spots. In: Criminology 29, S. 725-755. 
Sampson, R.J. (2006): How does Community Context Matter? Social Mechanisms and the Explanation of Crime Rates. In: Wikström, P.-O.H./Sampson, R.J. (Hg.), Crime and Its Explanation: Contexts, Mechanisms and Development. Cambridge: Cambridge University Press, S. 31-60.

Sampson, R.J. (2009): Disparity and diversity in the contemporary city: social (dis)order revisited. In: British Journal of Sociology 60(1), S. 1-31.

Sampson, R.J. (2012): Great American City. Chicago and the Enduring Neighborhood Effect. Chicago: Chicago University Press.

Sampson, R.J./Morenoff, J.D./Gannon-Rowley, T. (2002): Assessing „Neighborhood Effects": Social Processes and New Directions in Research. In: Annual Review of Sociology 28, S. 443-478.

Sampson, R.J./Morenoff, J.D./Raudenbush, S.W. (2005): Social Anatomy of Racial and Ethnic Disparities in Violence. In: American Journal of Public Health 95(2), S. 224-232.

Sampson, R.J./Raudenbush, S.W./Earls, F.J. (1997): Neighborhoods and Violent Crime: A Multilevel Study of Collective Efficacy. In: Science 277, S. 918-924.

Sampson, R.J./Raudenbush, S. W. (1999): Systematic social Observation of Public Spaces: A New look at Disorder in Urban Neighborhoods. In: American Journal of Sociology 105(3), S. 603-651.

Sampson, R.J./Raudenbush, S. (2004): Seeing Disorder: Neighborhood Stigma and the Social Construction of Broken Windows. In: Social Psychology Quarterly 67(4), S. 319-342. Sampson, R.J./Wikström, P.-O.H. (2008): The social order of violence in Chicago and Stockholm neighborhoods: a comparative inquiry. In: Kalyvas, S./Shapiro, I./Masoud, T. (Hg.), Order, conflict, and violence. Cambridge: Cambridge University Press, S. 97-119. Sariaslan, A./Langstrom, N./D'Onofrio, B./Hallqvist, J./Franck, J./Lichtenstein, P. (2013): The impact of neighbourhood deprivation on adolescent violent criminality and substance 
misuse: A longitudinal, quasi-experimental study of the total Swedish population. In: International Journal of Epidemiology 42(4), S. 1057-1066.

Savolainen, J./Lehti, M./Kivivuori, J. (2008): Historical Origins of a Cross-National Puzzle, Homicide in Finland, 1750 to 2000. In: Homicide Studies 12(1), S. 67-89.

Schwind, H.-D./Ahlborn, W./Weiß, R. et al. (1978): Empirische Kriminalgeographie:

Bestandsaufnahme und Weiterführung am Beispiel von Bochum. Wiesbaden:

Bundeskriminalamt.

Sessar, K. (1997): Zu einer Kriminologie ohne Täter oder auch: Die kriminogene Tat. In: Monatsschrift für Kriminologie und Strafrechtsreform 80(1), S. 1-24.

Sharkey, P. (2010): The acute effect of local homicides on children's cognitive performance. In: Proceedings of the National Academy of Sciences 107(26), S. 11733-11738.

Sharkey, P./Sampson, R.J. (2010): Destination effects: residential mobility and trajectories of adolescent violence in a stratified metropolis. In: Criminology 48(3), S. 639-681.

Sharkey, P./Faber, J.W. (2014): Where, When, Why, and For Whom Do Residential Contexts Matter? Moving Away from the Dichotomous Understanding of Neighborhood Effects. In: Annual Review of Sociology 40(1), S. 559-579.

Shaw, C./McKay, H.D. (1969 [1942]): Juvenile Delinquency and Urban Areas. Chicago: Chicago University Press.

Sherman, L.W./Gartin, P.R./Buerger, M.E. (1989): Hot spots of predatory crime: Routine activities and the criminology of place. In: Criminology 27(1), S. 27-55.

Skogan, W.G. (1990): Disorder and Decline. Crime and the Spiral of Decay in American Neighborhoouds. New York: Free Press.

Skogan, W.G. (2015): Disorder and Decline: The State of Research. In: Journal of Research in Crime and Delinquency 52(4), S. 464-485.

Smith, W.R./Frazee, S.G./Davison, E. (2000): Furthering the integration of routine activity and social disorganization theories: small units of analysis and the study of street robbery as a diffusion process. In: Criminology 38(2), S. 489-523.

Steenbeek, W./Hipp, J.R. (2011): A longitudinal test of social disorganization theory: feedback 
effects among cohesion, social control, and disorder. In: Criminology 49(3), S. 833-870.

Taylor, R. (2015): Community Criminology. New York: New York University Press.

Thome, H. (2002): Kriminalität im Deutschen Kaiserreich, 1883-1914. Eine sozialökologische Analyse. In: Geschichte und Gesellschaft 28(4), S. 519-553.

Townsley, M. (2017): Crime Mapping and Spatial Analysis. In: Leclerc, B./Savona, E.U. (Hg.),

Crime Prevention in the 21st Century. Insightful Approaches for Crime Prevention Initiatives. Cham: Springer, S. 101-112.

Tseloni, A. (2006): Multilevel Modeling of the Number of Property Crimes: Household and Area Effects. In: Journal of the Royal Statistical Society: Series A 169(2), S. 205-233.

United Nations Office on Drugs and Crime (2013): Global study on homicide 2013. Trends, contexts, data. Wien: United Nations Office on Drugs and Crime.

van Dijk, J./Tseloni, A./Farrell, G. (Hg.) (2012): The International Crime Drop. New Directions in Research. Houndmills/New York: Palgrave Macmillan.

Wacquant, L. (2009): Punishing the Poor: The Neoliberal Government of Social Insecurity. Durham, NC: Duke University Press.

Warner, B.D./Wilcox Rountree, P. (1997): Local Social Ties in a Community and Crime Model: Questioning the Systemic Nature of Informal Social Control. In: Social Problems 44, S. $520-536$

Weerman, F.M. (2011): Delinquent peers in context: a longitudinal network analysis of selection and influence effects. In: Criminology 49(1), S. 253-286.

Weisburd, D. (2015): The law of crime concentration and the criminology of place. In: Criminology 53(2), S. 133-157.

Weisburd, D./Eck, J.E./Braga, A.A. et al. (Hg.) (2016): Place Matters. Criminology for the 
Twenty-First Century. Cambridge: Cambridge University Press.

Weisburd, D./Groff, E.R./Yang, S.-M. (2012): The Criminology of Place. Street Segments and our Understanding of the Crime Problem. Oxford: Oxford University Press.

Wickes, R./Hipp, J./Sargeant, E./Mazerolle, L. (2016): Neighborhood Social Ties and Shared Expectations for Informal Social Control: Do They Influence Informal Social Control Actions? In: Journal of Quantitative Criminology, S. 1-29.

Wikström, P.-O.H. (1991): Urban Crime, Criminals, and Victims. New York: Springer.

Wikström, P.-O.H. (2006): Individuals, Settings, and Acts of Crime. Situational Mechanisms and the Explanation of Crime. In: Wikström, P.-O.H./Sampson, R.J. (Hg.), Crime and Its Explanation: Contexts, Mechanisms and Development. Cambridge: Cambridge University Press, S. 61-107.

Wikström, P.-O.H./Oberwittler, D./Treiber, K./Hardie, B. (2012): Breaking Rules. The Social and Situational Dynamics of Young People's Urban Crime. Oxford: Oxford University Press.

Wilson, J.Q./Kelling, G.E. (1982): Fixing Broken Windows: The Police and Neighborhood Safety. In: Atlantic Monthly (March), S. 29-38.

Wodtke, G.T./Harding, D.J./Elwert, F. (2011): Neighborhood Effects in Temporal Perspective: The Impact of Long-Term Exposure to Concentrated Disadvantage on High School Graduation. In: American Sociological Review 76(5), S. 713-736. 